Sección central

(Q)

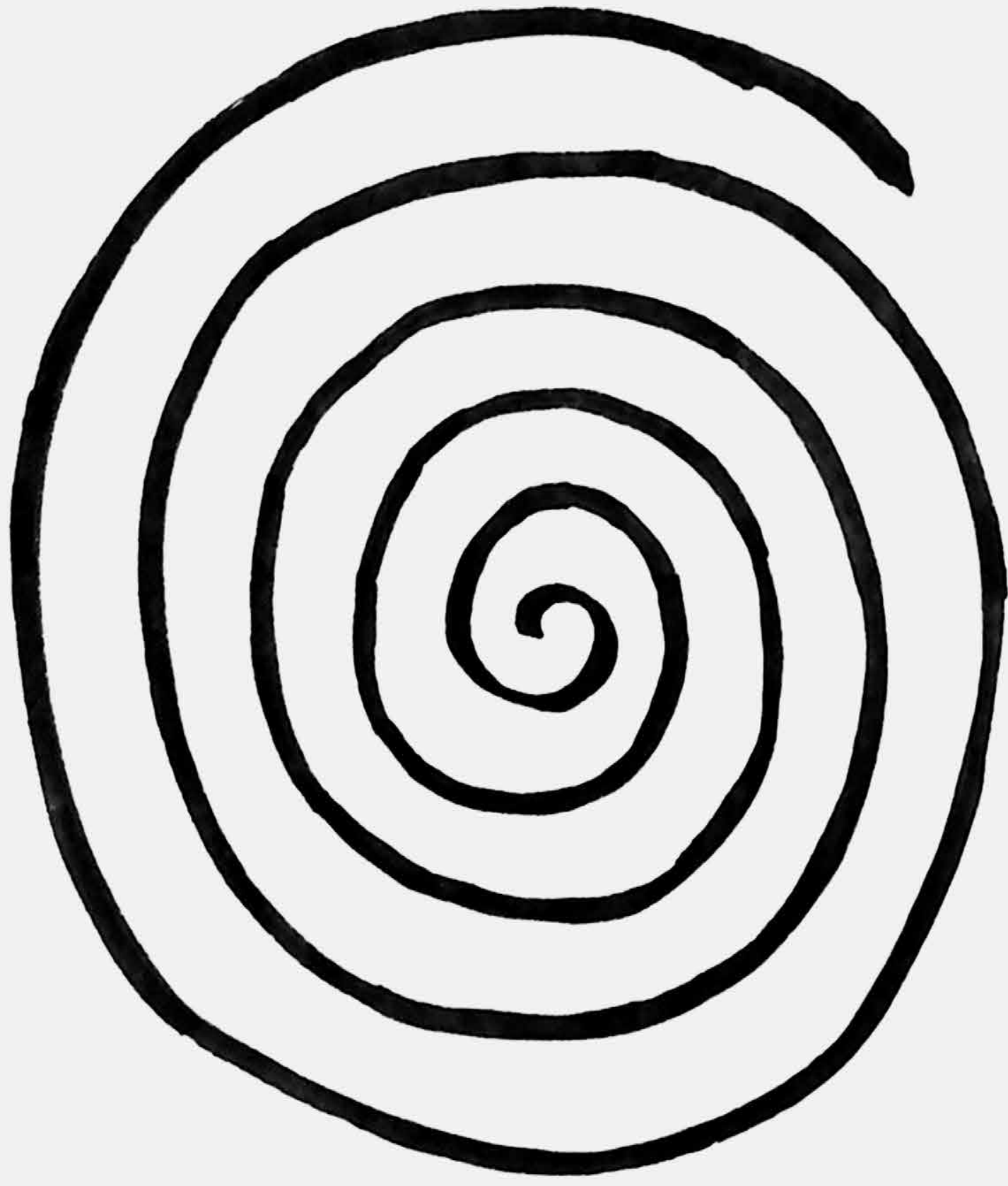

()) 


\section{Conocimiento y pluriversidad de mundos en universidades indígenas: Amawtay Wasi (Ecuador) y UAIIN- CRIC (Colombia)}

\section{Artículo de investigacion}

Recibido: 3 de septiembre de 2017

Aprobado: 18 de septiembre de 2017

Ramiro R. Huanca Soto

Universidad Mayor de San Andrés-UMSA

(La Paz, Bolivia)

ramiroreinaldo@yahoo.com

Cómo citar este artículo: Huanca Soto, Ramiro R. (2017). Conocimiento y pluriversidad de mundos en universidades indígenas: Amawtay Wasi (Ecuador) y UAIIN-CRIC (Colombia), Estudios Artísticos: revista de investigación creadora, 3 (3) pp. 137-149. DOI: https://doi.org/10.1448/ear. v3i3.12533

Trazos simbólicos en las clases de la UAIIN-CRIC, programa Pedagogía Comunitaria, 2015. Popayán. Fuente propia.

\section{Resumen}

La investigación que se presenta trata sobre las concepciones de conocimiento de dos universidades indígenas: Amawtay Wasi (Ecuador) y UAIIN-CRIC (Colombia). A partir de una investigación cualitativa de campo realizada en las comunidades y pueblos donde operan dichas universidades, se establece una interrelación de documentos curriculares y voces de sabios y sabias. El trabajo de campo ha permitido escuchar y poner atención en los lugares de enunciación desde donde hablan los sabios y sabias y cómo el conocimiento fluye desde sus perspectivas y experiencias. Se enfatizan dos ceremonias rituales, el Sakhelu (pueblo Nasa, Colombia) y el Raymi (pueblo Kichua, Ecuador), para dar cuenta de que el conocimiento está también en las prácticas culturales. En este trabajo fluyen las ideas de pluriversidad y pluriverso como nociones explicativas de la diversidad de mundos espirituales y la manera cómo pueden dialogar de manera intercultural con el conocimiento experto. El enfoque teórico es a partir de redes conceptuales provenientes del pensamiento decolonial e intercultural.

\section{Palabras clave}

Pluriverso, Pluriversidad, sabiduría, conocimiento, entramado, modernidad,

\section{Knowledge and Pluriversity of Worlds in Indigenous Universities: Amawtay Wasi (Ecuador) and UAIIN- CRIC (Colombia)}

\footnotetext{
Abstract

The present research article deals with the conceptions of knowledge found in two indigenous universities: Amawtay Wasi (Ecuador) and
} 
UAIIN-CRIC (Colombia). From a qualitative field research carried out in the communities and towns where these universities operate, an interrelation of curricular documents and the voices of wise men and women was established. The field work has allowed to listen and pay attention in the places of enunciation where wise men and women talk, and how knowledge flows from their perspectives and experiences. Two ritual ceremonies are emphasized: the sakhelu (Nasa people, Colombia) and the Raymi (Kichua people, Ecuador), to realize that knowledge is also to be found in cultural practices. In this work, the ideas of pluriversity and pluriverse flow as explanatory notions of the diversity of spiritual worlds, along with the manner in which they can dialogue in an intercultural way with expert knowledge. The theoretical approach is based on conceptual networks originating from decolonial and intercultural thinking.

\section{Keywords}

Pluriverse, pluriversity, wisdom, knowledge, network, modernity.

\section{Connaissance et pluriversité des univers dans les universités indigènes : Amawtay Wasi (Équateur) et UAIIN-CRIC (Colombie)}

\section{Résumé}

Le présent article de recherche porte sur les conceptions de la connaissance trouvées dans deux universités indigènes : Amawtay Wasi (Équateur) et UAIIN-CRIC (Colombie). À partir drune recherche sur le terrain qualitative, menée dans les communautés et les villes où ces universités fonctionnent, une interrelation entre les documents scolaires et les voix des hommes et des femmes sages a été établie. Le travail sur le terrain a permis d'écouter et de faire attention dans les lieux d'énonciation où les hommes et les femmes sages parlent, et comment les connaissances découlent de leurs perspectives et expériences. Deux cérémonies rituelles sont soulignées : le sakhelu (Nasa people, la Colombie) et le Raymi (Kichua, l'Équateur), pour comprendre que la connaissance se trouve également dans les pratiques culturelles. Dans ce travail, les idées de pluriversité et de pluriverse circulent comme des notions explicatives de la diversité des mondes spirituels, ainsi que la façon dont ils peuvent dialoguer de manière interculturelle avec la connaissance experte. L'approche théorique repose sur des réseaux conceptuels issus de la pensée décoloniale et interculturelle.

\section{Mots clés}

Pluriverse, pluriversité, sagesse, connaissance, réseau, modernité.

\section{Conhecimento e pluriversidade de mundos em universidades indígenas: Amawtay Wasi (Equador) e UAIIN-CRIC (Colômbia)}

\section{Resumo}

A investigação que se apresenta trata das concepções de conhecimento de duas universidades indígenas (Amawtay Wasi (Equador) e UAIINCRIC (Colômbia). A partir de uma investigação qualitativa de campo realizada nas comunidades e cidades onde operam tais universidades, se estabelece uma interrelação de documentos curriculares e vozes de sábios e sábias. O trabalho de campo tem permitido ouvir e prestar atenção em dois lugares de enunciação desde onde falam os sábios e sábias e como o conhecimento flui desde suas perspectivas e experiências. Enfatizam-se duas cerimônias rituais como o sakhelu (povo nasa, Colômbia) e o Raymi (povo Quichua, Equador), para dar conta de que o conhecimento está também nas práticas culturais. Neste trabalho fluem as ideias de pluriversidade e pluriverso como noções explicativas da diversidade de mundo espirituais e a maneira como podem dialogar de modo intercultural com o conhecimento experto. O enfoque teórico situa-se a partir de redes conceituais provenientes do pensamento decolonial e intercultural.

\section{Palavras-chave}

Pluriverso - pluriversidade - sabedoria - conhecimento - entramado - modernidade 


\section{lachai y Pluriversidad de mundos en Universidades Indigenas: Amawtay Wasi (Ecuador) y UAIIN-CRIC (Colombia)}

\section{Maillallachiska}

Kai tapuchi kauchikuna canchime kame isay Atun iachachii wasecunamanda paikunata uauai Amawtay Wasi (Ecuador) y UAIIN-CRIC. Tapuchei kallaresca alpape ruraska chi llagtape naipe maku atun iachachii wasi, kaiwa rurarenme trukai iachaikuna, parlo iachagkunata. Chi minga alpape uiarrkencheme y iachaicurrcancheme chi luarconape iachagkunata chi luarkonape iachagkata paikunata iachai iachachinkuna. Rurarenme iskai ailesrema, sakhelu (pueblo Nasa, Colombia) Raymi (Pueblo quechua, Ecuador) Chasa nengapa Nukanchipa iachaikuna kamkuname cada puncha ruranchame, chi minga Alpe llugseme suglla iuiai tukuikunapa uauaiwa, chi kawai kilakaska llugsenme iuaikunamanda colonial e intercultural.

\section{Rimangapa Ministidukuna}

Tukuikunapa, sabiduría iachii, conocimiento regsei, modernidad- musu.

\section{Para que la Madre Tierra no muera}

Para que la Madre Tierra no muera volvamos a danzar alrededor del Sol y de la Luna

las danzas del cóndor, la serpiente y el venado..

Dialoguemos en círculos en el día y en media luna en la noche. Hablemos en tiempo de ayer, de ahora y de mañana, con nuestros Yayas (abuelos), y nuestros Wawas (niños)..

Wankar Ariruma Kowii, poeta kichwa, Ecuador

\section{Introducción}

En el presente artículo se entreteje un entramado discursivo de sabios, sabias ${ }^{1}$ y documentos curriculares de las universidades indígenas Amawtay Wasi

\footnotetext{
$1 \quad$ Hay una diversidad de categorías propias de los pueblos ancestrales para nombrar las 'especialidades' de sabios, por ejemplo: amawtas, yachac, mayor, yatiri, chamakani, larama, the' wala, entre otras. En el presente artículo, se nombra en general con la categoría de sabio o sabia, inclusive a sujetos indígenas letrados o académicos. De sus voces entretejemos
}

(Ecuador) y UAIIN-CRIC (Colombia), a partir de un proceso metodológico convivencial en comunidades y pueblos sobre la base de conversaciones y acompañamientos en determinadas prácticas culturales y rituales como el Sakhelu (pueblo Nasa, Colombia) y el Raymi (pueblo Kichua, Ecuador). Ese entretejido responde a la siguiente pregunta: ¿Cuáles son algunos rasgos decoloniales ${ }^{2}$ que se evidencian en las universidades indígenas como una respuesta del sentir/pensar/hacer propio a la colonialidad del poder, del saber, del ser y la naturaleza? (Quijano, 1999). Dicho entramado tensiona la forma de estar en la subjetividad moderna, así como la idea universalista de conocimiento experto.

La Universidad Intercultural de las Nacionalidades y Pueblos Indígenas, Amawtay Wasi, fue creada en 2004 y reconocida legalmente por el estado ecuatoriano, por tanto, fue parte del Sistema Nacional de Educación Superior, mediante Ley de la República No. 2004-40, del 28 de julio del 2004. Su estatuto orgánico fue aprobado por el pleno del Consejo Nacional de Educación Superior -CONESUP, mediante Resolución RCP.S11 No. 35205, el 20 de noviembre de 2005. En el proceso de su creación, si bien se asumen todas las formalidades de una universidad perteneciente al sistema de universidades, también se instalan lógicas propias como la minka, concepto que alude al trabajo comunitario.

Es importante ver que en el ejercicio para la construcción epistemológica de la Universidad, varios fueron los partícipes, obedeciendo a la matriz colectiva de donde provenían: amawtas, mayores, hombres y mujeres, indígenas y no indígenas, que contribuyeron con sus criterios y saberes a la recuperación de categorías y a la configuración de una epistemología pertinente con los pueblos. En este sentido, la participación de científicos y humanistas, que fueron convocados a ser parte de una

\footnotetext{
el entramado de sus sabidurías, atendiendo a lo que dice la Amawtay Wasi: "†odos somos sabios en crisálida".

2 Lo decolonial es una opción de pensamiento y expe riencia que da la vuelta a formas de dominación en un otro sentido creador que va más allá de la descolonización. Instituye una forma de ser y estar desde acciones colectivas afirmativas, algo contrario de lo que ocurre en Bolivia donde la descolonización como discurso ideológico e instalado en la macro política es parte del Estado y poco o nada desde las comunidades y pueblos.
} 
demanda y reivindicación de los pueblos y nacionalidades del Ecuador, como es contar con una universidad que trabaje en las comunidades y pueblos.

Es importante ver que en el marco de su propia configuración la misión se asume como una minka: "Contribuir en la formación de talentos humanos que prioricen una relación armónica entre la madre naturaleza y el ser humano sustentándose en el buen vivir comunitario como fundamento de la construcción del Estado plurinacional y la sociedad intercultural. Adelantándose a los procesos de cambio que vivió el Ecuador, pues el 2004 no se asumía como Plurinacional ni Estado Intercultural como ahora se afirma en su Constitución Política, la Amawtay Wasi, es una de las primeras instituciones indígenas que plantea el tema del buen vivir o el Sumak Kawsay, lo que en Bolivia se asume como Vivir Bien/Suma Qamaña. Pero no sólo sería pionera a nivel del discurso constitucional planteando un horizonte civilizatorio posible, de ser construido como alternativa a un sistema civilizatorio que avasalla la naturaleza, sino a nivel de la organización de una sociedad donde se establezca las relaciones de convivencia desde la interculturalidad. Por ello el concepto de sociedad intercultural, muestra la propuesta indígena como una posibilidad no etnocentrista ni orientada sólo a los pueblos ancestrales milenarios, sino a toda la sociedad en general.

El planteamiento de sociedad intercultural es un elemento importante si se la asume en el contexto de las políticas de interculturalidad que planteaban los estados nacionales o republicanos, particularmente de Ecuador y Bolivia. Las reformas educativas de estos países si bien desde la década de 1990 enfatizaron ciertas proyecciones de educación intercultural bilingüe y la necesidad de implementar la interculturalidad como política de Estado, siempre fue pensada y proyectada sólo hacia sectores indígenas, excluyendo de las relaciones interculturales a otros sectores importantes como las identidades no indígenas y urbanas. Sólo a partir de las reformas profundas que acontecen desde hace 10 años, aproximadamente, tanto en Bolivia como en Ecuador, las políticas de interculturalidad se asumen como una política universalizada a toda la sociedad y no sólo a ámbitos rurales o sobre identidades exclusivas.
En la formulación de su Visión, acogiéndose a la forma de gestión de las universidades públicas, la Amawtay Wasi la asume como sueño, planteando que se trata de "El sueño de recuperar el tejido vivo que entretejemos en la interculturalidad cósmica" (Sarango, 2008, p. 8). La mirada de gestión está puesta en la cosmovisión propia a partir de pensar una realidad fundada en el tejido vivo. Pero más, se trata de la existencia de una colectividad donde la práxis de tejer no está fundada en la individuación sino en la comunidad, por ello la afirmación de "entretejemos". Esta acción colectiva no es sólo motriz o funcional para un acto de imbricaciones creadoras de un tejido material, sino que es la metáfora simbólica que explica la realidad a la que una persona va contribuyendo a hacerla posible de manera colectiva. En este sentido, el entretejido colectivo implica tejer la interculturalidad cósmica, una noción que articula las relaciones de convivencia con la naturaleza y el cosmos.

La interculturalidad, por tanto, no se asume sólo a partir de las relaciones entre seres humanos, la sociedad intercultural que fundamenta la Amawtay Wasi, se asume también en relación con el tejido vivo de la naturaleza y el cosmos, ámbitos que en las políticas estatales de Ecuador y Bolivia no serían sino consideradas después del 2009-10, momento constitutivo y fundacional del "proceso de cambio" en Bolivia y la "Revolución Ciudadana" en Ecuador.

Por su parte, la Universidad Autónoma Indígena Intercultural del Consejo Regional Indígena del Cauca (Colombia) creada en 2003, es una entidad que articula toda una historia organizativa de luchas y reivindicaciones por más de 40 años. Entre sus ideales está la búsqueda colectiva de una educación propia para los pueblos indígenas. La educación propia se asume como una educación pertinente a la visión, situación cultural, necesidades y proyección de las comunidades locales. Estas comunidades locales y pueblos han sido parte de un proceso donde cada localidad viene construyendo colectivamente su Plan de Vida. ${ }^{3}$

\footnotetext{
3 El Plan de Vida es un plan de cada pueblo, elaborado colectivamente y según su horizonte ancestral. Este Plan de Vida está generado en el marco de la autonomía territorial de cada pueblo
} 
Cada Plan de Vida de cada pueblo, de los trece pueblos que habitan en El Cauca, ha sido promovido en su construcción colectiva a partir de la red organizada del CRIC, organización vigente desde 1971. Cada Plan de Vida expresa la subjetivación colectiva de cada pueblo y en muchos casos se opone a los planes y diseños nacionales y globales del Estado colombiano. En todos estos años el Plan de Vida de cada pueblo se ha constituido en una herramienta de resistencia y re-existencia. Por un lado se resiste los embates coloniales de las políticas nacionales y por otro se re-existe en cuanto reconstituye sus propias prácticas de vida en continuidad a la memoria ancestral prehispánica. Si bien toda experiencia y prácticas de vida están cruzadas por relaciones de dominación, en toda re-existencia propia emerge la reconstitución en tensión con elementos dominantes, y no por ello también formas fluidas de códigos, lenguajes, prácticas plenamente propias.

Es en este marco que inscribimos la propuesta del presente artículo.

\section{El conocimiento y la subjetividad moderna}

Hay una constelación de intelectuales europeos que diseñaron el pensamiento de la modernidad a nivel planetario. Pese a que los griegos no representan el inicio de esa constelación, pues los africanos, los árabes, los persas, los indios, los chinos aportaron mucho al universalismo europeo, la hegemonía de sus teorías desde diversas ramas del conocimiento se diseminó a medida que el capitalismo se expandía. Una de esas teorías, es el "Dasein" que Martín Heidegger planteó como una forma de estar en el mundo. Para Mignolo (2014), esta forma de estar implica al sujeto moderno y fue proyectada como perspectiva universal, por tanto imperial. "Por ejemplo el Dasein, es una manera de pensar y estar en el mundo que surge de la subjetividad moderna, no de la subjetividad colonial (sujetos colonizados) y en procesos de liberación, descolonización." (p. 62). Esta propuesta se universaliza por medio del saber institucionalizado que subjetiviza, separa y avasalla las experiencias de ser y estar de pueblos ancestrales milenarios.

\section{Conocimiento para dominar y separar}

La política imperial y la subjetividad moderna han impregnado los sistemas educativos en continuidad al avance del capitalismo y la ciencia. La producción moderna de conocimiento si bien demuestra muchos adelantos técnicos y tecnológicos, sociales y humanísticos, no deja de estar subordinada a una idea y praxis del poder, pues en el marco del saber moderno, no se conoce para cambiar y transformar al mundo, sino

Para dominar, para someter, para subordinar, para controlar. La forma cómo se enseña, cómo se piensa y cómo se define lo Real, es decir lo existente en cuanto tal, rebasa los límites epistemológicos y metodológicos del conocimiento y se convierte en un asunto político que tiene que ser debatido y comprendido desde la política porque implica al poder. (Dávalos, 2013, p. 23).

El conocimiento tiene implicaciones políticas porque reproduce la colonialidad del poder expresada en la división y explotación del trabajo, la propiedad privada y las relaciones de producción introducidos desde la invasión colonial (Quijano, 2001). Poder y conocimiento, entonces, implican a la realidad, a la política y a la economía y de acá su dominio estructural expandido a nivel global sobre los estados e instituciones del saber. El dispositivo de este dominio se sustenta en las relaciones de inferiorización racial y para legitimarse requiere de un tipo de conocimiento que naturalice la explotación capitalista y centralice una política de "sucesivas separaciones o particiones del mundo de lo real que se dan históricamente en la sociedad occidental" y "la forma en que se articulan los saberes modernos con la organización del poder, especialmente las relaciones coloniales/imperiales del poder constitutivas del mundo moderno" (Lander, 2005, p.5). A partir de estas separaciones se demarcará la línea abismal y las injusticias cognitivas con los saberes y conocimientos sociales, particularmente de los pueblos ancestrales milenarios. Asimismo, si no se conoce para transformar entonces el conocimiento está separado de lo real y en todo caso políticamente de las comunidades y pueblos. Las sucesivas separaciones no se refieren solamente a la enemistad de las disciplinas (en duras y blandas) operadas desde el siglo XVIII, según Wallerstein. Se refieren también a las 
separaciones cognitivas entre universidad y pueblo, por tanto a la injusticia cognitiva que precede a la injusticia social global (De Sousa Santos, 2010).

En este contexto y desde la UAIIN-CRIC y Amawtay Wasi, se trata de establecer una profunda relación entre universidad indígena y entretejido de sucesivas continuidades de formas de conocer con los pueblos y comunidades (no sus separaciones) que implican el desprendimiento de las relaciones de ser, conocer, hacer y con-vivir con la naturaleza y las leyes de la Pacha (Huanca, 2015) en una idea y praxis otra de poder (Walsh, 2009).

\section{Conocimiento para sembrar la vida}

En tal contexto, las universidades indígenas exponen algunos rasgos decoloniales desde sus experiencias respecto a la colonialidad del poder, del saber, del ser y la naturaleza. Una de esas experiencias convoca al yachak, Jaime Pilatuña, de la nación Kitukara, sabio docente de la Amawtay Wasi:

Tenemos que escuchar a nuestros primeros padres antiguos, la luz de sabiduría de nuestros mayores y abuelos, ellos nos dan claridad, ayudan a caminar. Ellos aprendieron a conocer la naturaleza y el cosmos, hablaron con las nubes y danzaron en ofrenda a las semillas para que germine bien alegre y haya buenas cosechas. Nuestros mayores sabían leer y conversar con las estrellas y guiarse por las influencias de las fases de la luna. Leían los libros de la naturaleza, si se puede decir. (Conversación, 4 de marzo de 2016.).

El yachak Pilatuña señala la necesidad de la escucha para caminar y que los primeros padres, como seres ancestrales de millones de años pueden dar claridad. Habla de los libros de la naturaleza y la manera de aprender a leer y conversar con las estrellas, pues la naturaleza es sujeto de lenguaje, a contracorriente de la filosofía del lenguaje (Navia, 2002) que enfatiza sólo en el ser humano las propiedades de escuchar y conversar.

En esta línea de pensamiento la UAIIN-CRIC en su documento Sistema Educativo Indígena Propio, plantea:

Los mayores han dicho que el Proyecto de Vida se ha ido construyendo a partir de lo que vive y lo que siente, para satisfacer la vida misma, o sea para llenar unos vacíos o necesidades que se tienen, y los

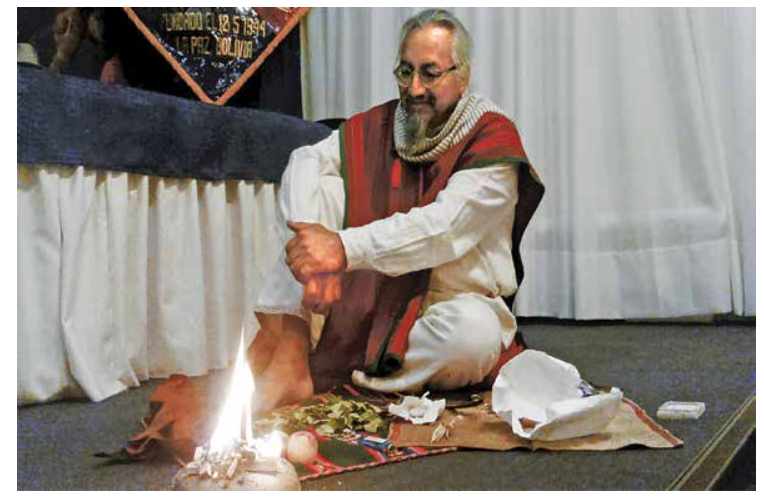

Yachak Jaime Pilatuña del pueblo Kitukara, sabio de la Universidad Intercultural Amaytay Wasi, en el Auditorio de la Casa Marcelo Quiroga Santa Cruz de la Facultad de Humanidades y Ciencias de la Educación. Visita realizada en marzo de 2016. Fuente: propia.

otros aspectos que se tienen que ir fortaleciendo y dentro de eso la más sentida y la más vista ha sido tener primero el territorio, porque para un indígena sin territorio no hay nada. Mientras está, hay vida, hay cultura, porque estamos sembrando la vida en la parte espiritual y la parte material (SEIP, 2011, p. 47).

Es importante ver que lo que "han dicho" los mayores emerge y se desparrama como mandato oral desde una racionalidad fundada en lo que se vive y se siente. El Plan de Vida orientado "hacia la vida misma" implica la forma de estar en el sustrato material político y económico. Político porque el conocimiento se genera y retorna a la forma de estar y ser en comunidad en una dimensión social, como es la de emancipar o liberar de las dominaciones extrañas así como revitalizar su propia continuidad histórica. Económico porque se trata de estar en el territorio para "Ilenar unos vacíos o necesidades que se tienen", es decir, que el Plan o Proyecto de Vida implique satisfacer las necesidades vitales de la vida.

La acción de la siembra dual como forma desprendida de la subjetividad moderna, es la acción imposible para la ciencia euro-norte-occidental, que ha expandido su ocupación experimental como una forma de estar sobre la materia, desalojando la estancia de la parte espiritual. "Los mayores han dicho" sobre la necesidad de reproducir un sistema de vida propio fundado en la complementariedad de la dualidad material y espiritual, no en 
su separación. Como decía el sabio Viluche en sus clases:

Para ello hay que estar sembrando en el territorio habitando un pluriverso de mundos (Escobar, 2014). ¿Qué significa estar en el territorio, sembrando la parte espiritual y la parte material?

Para el pensamiento indígena, la naturaleza o el mundo es un ser espiritual y material en íntima relación, por eso, en el caso de la cultura Nasa el territorio es concebido como una casa, en tanto que espacio y semilla; por ser embrión de la vida, la naturaleza es pues, una madre que genera vida. (Maya, 2014, p. 25) cita a Cxayu'ce (1999)

La íntima relación del ser espiritual y material de la naturaleza descentra la concepción antropocéntrica de la filosofía abstracta. La intimidad implica un estar e interaccionar juntos, y la forma de estar implica habitar la dinamización de esa intimidad. Por ello la importancia de saber estar y sentir el territorio, no sólo como medio ambiente, recurso natural o suelo productivo, tal como lo definen los discursos materialistas y desarrollistas. Habitar no es un estar estático, contemplativo o tener bajo control y dominio, es un movimiento interno del cuerpo que se despliega en intenciones prácticas, afectivas y cognitivas que hace despertar y parir la condición dual de la vida material y espiritual.

La siembra toma impulsión en la semilla sembrada para cuidar la vida y cuidarla también políticamente cuando ese sentido dual de la siembra está amenazada con la privatización de la tierra, del agua, la contaminación del aire, el extractivismo colonial de las energías, el uso de transgénicos, es decir, cuando las geopolíticas de conocimientos y la ciencia separa las partes material y espiritual. Como dijo la mayora nasa, Dolly, en un trueque que compartimos en Quintana, cerca al pueblo Kokonuko:

Hay que hacer despertar a las semillas y hablar y danzar con sus espíritus. Las semillas se despiertan con la música, la chicha y cuando bailamos con ellas. Hay que saber pedir permiso a los espíritus del territorio y de arriba y de abajo y de aquí pa' allá. (Conversación, 15 de diciembre de 2014).

No se trata sólo de investigar en laboratorio, que puede ser la parte material, tal cual hacen los pueblos del Cauca en su política educativa, como el pueblo Nasa a través del CEFIC y su Propuesta

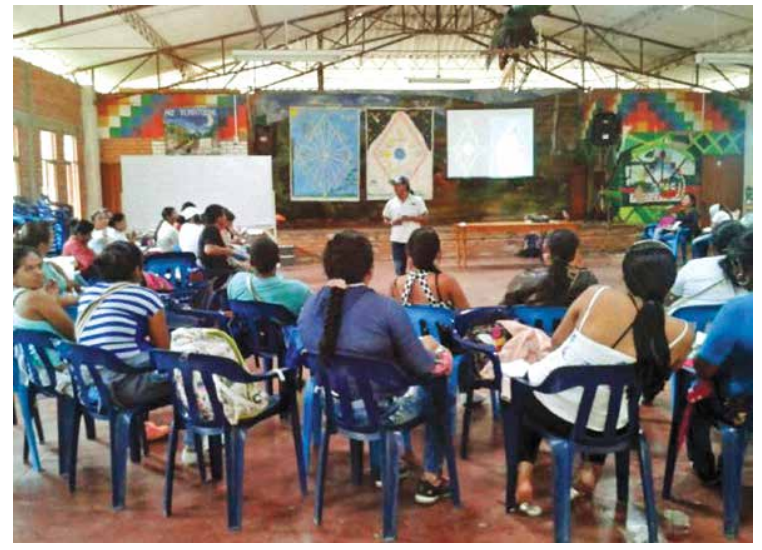

Sabio Joaquín Viluche, en una clase sobre el camino del sol y la luna. Popayán, 2015. Fuente: propia.

Pedagógica del área Naturaleza y Sociedad (2007), además, danzar junto a las semillas, las cuales están relacionadas con una totalidad de seres espirituales. Por ello es importante la ceremonia del Sakhelu en tanto práctica de fertilidad y reciprocidad. En este acto ritual que se realiza en septiembre, se dinamizan el sol, la luna, las estrellas; el trueno, el viento, el arco iris; las montañas, los ríos, los árboles, las plantas medicinales y el colibrí, a partir de la danza con las semillas. La comunidad en minga se re-articula para generar ese espacio del despertar de las semillas. Cada cuerpo comunero asume su corporalidad porosa y permeable pues se vive la experiencia de un cuerpo interconectado a las semillas, a los seres espirituales del territorio, al cosmos. No existe la separación entre el tiempo y espacio ni el exterior objetivo y el interior subjetivo (Marcos, 2010). Es una danza envolvente de la reciprocidad colectiva donde los cuerpos son parte de un fluido, por lo que las oposiciones binarias están ausentes. Naturaleza, cultura, razón, sentimiento, acción y política confluyen como dice el sabio Manuel Sisco, "en una cosmoacción integral de la comunidad," (Conversación, 17 de diciembre de 2014).

En esa cosmoacción los espíritus tendrían relación con el contexto sistémico de la naturaleza y el cosmos, mucho más en una ceremonia, pues la experiencia espiritual de un ritual "no es cualquier cosa, es el momento en que uno se renueva, es como salir nuevito, te regenera, es como un aire 


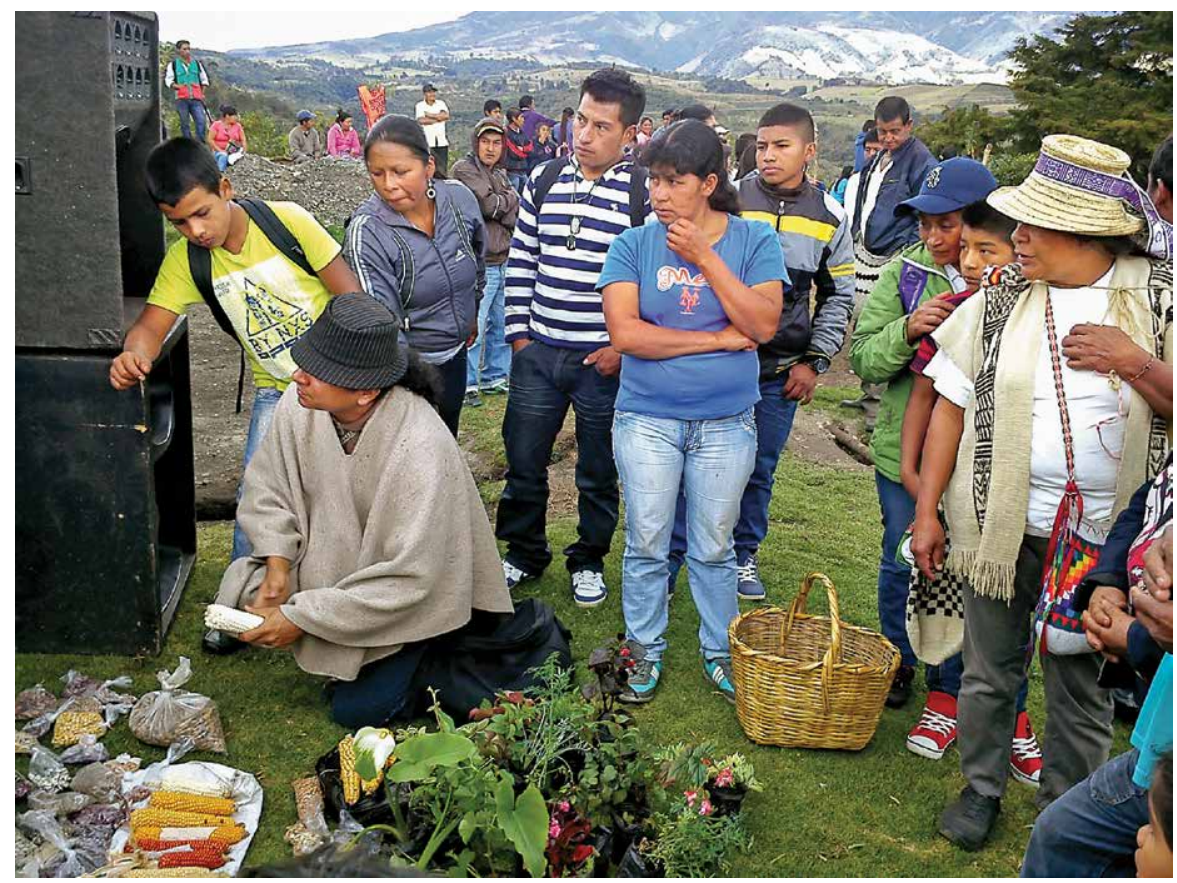

Sabia Dolly conversando sobre las semillas en el trueque de Quintana, pueblo de Kokonuko. Fuente: propia.

puro que se derrama sobre vos. Otro siempre sales y al hablar con la Pachamama te conoces (...)" (Conversación, 2 de febrero de 2015). El sabio Gonzalo, se refería a la nueva y otra condición humana que genera a la naturaleza como sujeto de lenguaje, para el autoconocimiento en un acto ritual y de qué manera en la generación del saber se implica el ajayu (espíritu). La regeneración es una experiencia que genera otra humanidad, otra experiencia del cuerpo conectada a otro orden; a diferencia del cuerpo que se resiste y se impermeabiliza, el cuerpo implícito señalado por el sabio se muestra poroso y abierto a otra condición espiritual. Ese "aire puro que se derrama" tiene analogía con el derrame fecundo del agua sobre la tierra y del cuerpo como lugar permeable de flujos de las fuerzas gravitacionales de la comunidad y el territorio. Esa experiencia permite entrar en una condición y mutar en otra. Es la conversión profunda de una forma trascendente de fluir entre estar y ser. Como dice Arnold y Yapita (2000) citando a Elvira Espejo, respecto a la piel amerindia:
La piel es un mediador entre lo interno y lo externo, un "cernidor" (suyuña) con poros abiertos (sit'artata) que se debe proteger. De este modo, la condición de la piel, y las envolturas de pieles adicionales en textil, definen la condición de ser persona y, todavía la del cuerpo social.

La noción amerindia de la piel, como del cuerpo mismo, en su calidad de mediador poroso entre los órganos internos y el mundo externo, nos da pautas acerca del pensamiento andino sobre los diferentes espíritus del cuerpo (ajayu, janayu, animu, suti, entre otros.) (p. 203).

\section{Conocimiento desde el 'pensar fluido'}

Se conoce para dominar, y esa finalidad ha avasallado pueblos, lenguas y culturas, la UAIIN-CRIC postula dar la vuelta a ese proceder y comenzar a conocer para tejer. Esta finalidad no es abyecta de la condición colonial, al contrario, se teje precisamente para no caer en el saber y poder moderno de someter, subordinar y controlar. Aunque no se conocieron Dávalos y la abuela del th'e wala, Ángel 
María, en un periodo discursivo del PEBI se coincide en que el saber moderno es distinto al saber del pueblo Nasa. Th'e wala, dijo lo siguiente:

Como mi abuela era tan furiosa, ella decía: ¿Qué están aprendiendo ustedes? —Decía- ¿No están aprendiendo ustedes a ser exagerados y a ser brutales con la gente? —Decía-. Nuestra forma de vida no es tal. Y ella preguntaba eso hasta Ilorar. Decía: 'Nosotros no queremos esas actitudes, no quere mos eso.' A ella le enfadaba que tanto tiempo que llevan con la educación, la palabra nuestra (el pensamiento) no le hayan enseñado y mucho nos encomendó y pensábamos: '¿De qué será que nos habla? ' Esa vieja lo que exigía era que se enseñara lo nuestro. Ella anhelaba que se enseñara lo que es de nuestra vida (...) Nosotros los nasa solíamos pensar tan fluidos como el agua(...)". (PEBI-CRIC 2004, p. 98).

Hay un modo de ser expresado en un elemento que no sólo se bebe, sino se piensa. El pensar tan fluidos convoca a todas las vertientes de pensamiento. Es una metáfora líquida que subvierte la mente seca y estancada del pensar descontextualizado y cuerpo anatómico, no relacionado; para este pensar fluido se requiere de sonoridades ancestrales, por tanto, el ritmo, el sonido, el canto, la música, las frutas y la comida en abundancia preceden al pensar fluido.

En este trance se re-establece el equilibrio con el pluriverso de mundos relativizando toda estratificación cognitiva. El pensar fluido rompe la idea del pensar como recipiente del saber. La mente no recepciona contenidos, son el cuerpo y la mente abiertos a un fluir recíproco con la totalidad de seres espirituales.

Pensar como el agua implica a los afluentes de los mundos heterogéneos del pluriverso contra la brutalidad epistemológica de pensar y tratar con arrogancia a la gente. Se trata de un hilo hidráulico de sangre que circula "dentro del cuerpo y también fuera del cuerpo, uniendo en su curso a los cuerpos de las personas que están en contacto las unas con las otras" Reniifo (2012) citando a Belaunde (2008, p. 68). "No queremos esas actitudes", decía la abuela del th'e wala, poniendo en relación viviente el pensamiento propio, la construcción de la subjetividad y la acción ética y fluida con el otro. Lo que ha dicho la abuela del th'e wala, en este sentido, supera el binarismo racionalista, yo-otro, cuando las disposiciones y comportamientos del cuerpo se apuntan "hacia el corazón de la alteridad." (Rizo, 2015, p.19).

\section{Conocimiento y revivencialización en el Raymi}

La Amawtay Wasi, asume una concepción de conocimiento que descentra el reino de la mente, el saber discreto y abstracto, "el saber sin danza, medio aburrido", como decía el sabio, Gerardo Simbaña, (Conversación, 20 de enero de 2017), cuando dialogábamos sobre la espiritualidad del Aya Uma en las fiestas del Inti Raymi en Quito. En este trance la pluriversidad postulada en su currículum no es una formalidad académica y fría, sino más bien tiene el sentido de ser una experiencia convivencial simbólica en las comunidades. Al respecto, es necesario compartir las autoafirmaciones reconstitutivas del ser en ese proceso de entender la pluriversidad no sólo como categoría externa al runa (persona íntegra en kichua) o como herramienta ortopédica que permita objetivar y realizar interacciones racionalizadas y discretas. Al contrario, la pluriversidad implica una vivencia, un acto fluido de lo inmanente del cuerpo a la totalidad trascendente de los mundos de los seres espirituales.

Benigno Shingre, indígena Saraguro, docente de escuela primaria y secundaria, titulado de la Amawtay Wasi, da cuenta de ese proceso reconstitutivo de su sami (espíritu en kichua) en función a la pluriversidad reconstituida por medio de la pedagogía concreta y con-vivencial:

Eso ha sido, para mí al menos, fundamental y me siento orgulloso de ser de la Amawtay Wasi y orgulloso también de haber recibido todo un bagaje de experiencias, de conocimientos, que me han servido para ir construyéndome en el proceso de esta nueva vida, y un poco también, yo desde antes hacía la parte espiritual, me ha ayudado a sustentar la parte espiritual, yo, bueno un poco, empecé a hacer de yachak o tayta, desde muy joven, bueno yo tengo rasgos también; vengo de una familia de padres, abuelos, tatarabuelos que han sabido de la medicina ancestral, pero desde la clandestinidad, y bueno en el año 85 muy jóvenes, hacíamos danza, música, y ahí empezamos con el profesor la reconstrucción, revivencialización del Raymi. (Conversación, 12 de diciembre de 2014).

El orgullo de Shingre al ser de la Amawtay Wasi, radica en el proceso de reconstrucción de su experiencia y memoria como Saraguro. Que la Amawtay Wasi le haya ayudado a sustentar su parte espiritual, y a fortalecer lo que traía de niño y de joven, tanto de la medicina como de la música 
y la danza, muestra el horizonte distinto de otras universidades orientadas a "desindianizar" la identidad originaria, es decir a blanquear al indio o al negro, a convertirlo en un profesional confeso de alguna religión extraña o de convertirlo en un científico frío, objetivo y eurocéntrico.

Shingre, se fue mutando desde adentro y acercando a la resolución de su contradicción, "construyendo en esta nueva vida", haciendo lo que el mundo Saraguro implicaba, no lo que del mundo euro-norte-occidental se replicaba, aunque hacía uso de muchos de sus elementos. En ese proceso había una tensión entre "el desprendimiento del mundo ajeno, y la recepción simultánea de la vitalidad consciente de su identidad y cultura" (Smith Colque, conversación, 11 de septiembre de 2014). Esa vitalidad consciente acompañada también de intelectuales naturales de la comunidad:

(...) empezamos con el Inti Raymi, muy folclóricamente, muy, cómo diría yo, muy chabacanamente, muy, cómo te decía, muy superficialmente, yo hacía danza, otros cumpas hacían música y empezamos así y yo desde entonces hacía la parte de espiritualista, cómo le llaman en Bolivia, al tayta, al chamán; bueno, aquí se ha dado y se hace todo más con el yachak y con el tayta, más ya, entonces eso empecé a hacer, bueno, a veces fuerte para mí, porque no estuve preparado a veces, me iba nomás de un ambiente a otro ambiente y yo ya había hecho mis energéticos y así fui avanzando. La Amawtay Wasi me hizo que sostenga esa parte, que sistematice, y con eso un poco como que hacen todos los cimientos para sustentar la parte espiritual." (B. Shingre, entrevista 10 de enero 2014).

Shingre, transita por momentos blandos, débiles, a los que llama "chabacanamente", "superficialmente" hasta llegar a vivencias "fuertes" ayudado "en" la experiencia del tayta y del yachak. Y estar en dicha experiencia implica estar en y con el mundo de las autoridades espirituales. Sin embargo, aún no establecido plenamente en el mundo propio, deambula de un lugar a otro, haciendo "mis energéticos" y así va avanzando en el camino de recuperación de su interioridad espiritual. El estar avanzando, por tanto, no sólo se da en el orden de relación con el otro, por ejemplo, con el mestizo, sino en estar avanzando en la profundidad interior del ser originario. En este caminar que va de la experiencia superficial, blanda y débil a la experiencia significativa, dura y fuerte de la descolonización espiritual, la Amawtay Wasi ha tenido su efecto práctico en la vida misma de Shingre. La Amawtay Wasi habría operado en su formación profesional, no sólo en su formación cognitiva o de conocimientos mediados por la interculturalidad, al manejar las dos matrices de conocimientos -occidental y originario- sino porque habría sostenido la parte sustancial de su base existencial, los "cimientos" necesarios para la reconstrucción del Samay (espíritu ancestral).

\section{Conocimiento y tejido}

Es importante la generación del conocimiento desde acciones concretas y en situaciones de reciprocidad colectiva. Para la Amawtay Wasi, en estas instancias es cuando se activan las comunicaciones simbólicas, la expresión de los seres espirituales por medio de símbolos.

En la UAIIN-CRIC, de igual manera, el conocimiento está en otras dimensiones que trascienden el aula y al edificio cerrado y sus procesos abstractos (des) relacionados del territorio y la comunidad. Por ello, está fundada en el pensar, andar y existir desde, con y para los pueblos, al plantear que:

Desde el pensamiento de nuestros pueblos, la memoria colectiva es vivir tejiendo el pensamiento de los antepasados, es el tejer diario de las personas que habitamos el territorio. Este ejercicio de permanecer pensando, es el acto de reflexionar sobre los sucesos mientras vivamos. Ello permite que se continúe construyendo la memoria colectiva de los que nos antecedieron y por lo tanto esta memoria colectiva de los que nos antecedieron contribuye a la consolidación de una historia vivida y sentida desde nuestra propia realidad (SEIP, 2011, p. 33).

La memoria colectiva es la acción de experiencia y vivencia autoconstituida. Una acción de vida que anuda el pensamiento de los antepasados, no como algo antiguo que puede ser objetivado a la distancia temporal, ni como rescatar un fósil arqueológico, sino como un "tejer diario de las personas que habitamos el territorio".

Vivir tejiendo el pensamiento de los antepasados" implica hacerlo en cuanto "habitamos el territorio". Aquí subyace el carácter temporal y espacial del conocimiento, pues no se trata de descubrir o generar cambios en la naturaleza o la sociedad por el uso intensivo de la acción en cualquier momento y cualquier lugar sobre el objeto de estudio en una relación de sujeto versus objeto. Se trata de anudar 


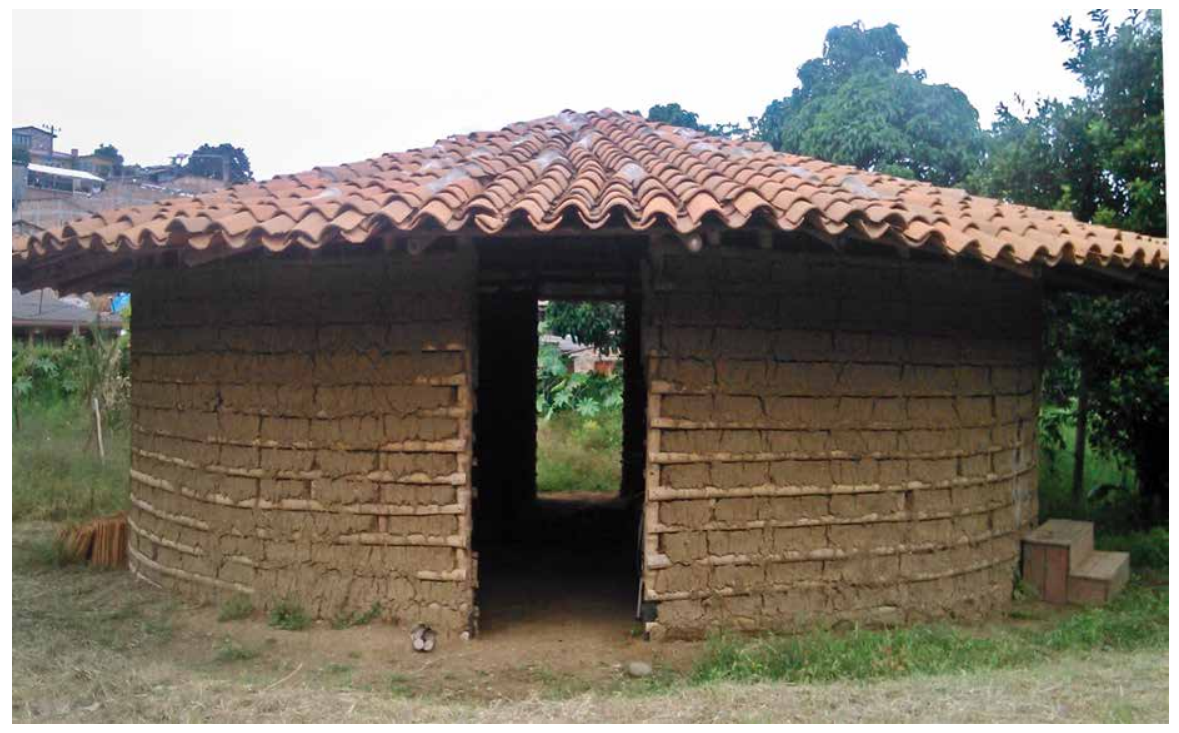

Un centro de aprendizaje o "aula" de acuerdo a la Chakana y según la entrada y salida del sol. Casa ancestral Yanakona. "No se requiere mucha infraestructura sino lo necesario y salir a caminar la palabra". Fuente: propia.

e imbricar el pensamiento como una tarea ética que articula el pasado con el presente, una forma ampliada del qhip nayra ${ }^{4}$ y sobre un espacio concreto como es el territorio.

La acción de "permanecer pensando" como acto de reflexión en viva experiencia localizada en lugar, descentra la mirada ocular céntrica del conocer por medio de la centralidad de la mente. Se trata de "reflexionar sobre los sucesos mientras vivamos". La vivencia entraña al cuerpo, a la acción, a las dimensiones sensoriales y signos corporeizados en señas, mandatos internos sintientes y visionarios como los sueños así como la praxis ritual ceremonial e historia viva acumulada previamente. No es un acto espontáneo y de libre disposición cognitiva, como quien hecha a funcionar la mente rápidamente y en cualquier lugar, como quien entra a un laboratorio o se sienta frente al computador, tal cual es la política moderna de

\footnotetext{
$4 \quad$ Qhip Nayra es una categoría aymara trabajada por el Taller de Historia Oral Andina-THOA, que implica la multidimensionalidad del tiempo-espacio en el sentido en que el pasado está adelante y el futuro está atrás.
}

conocimiento de los Estados plurinacionales de Bolivia y Ecuador (Villavicencio, 2013), al crear ciudadelas de conocimiento para producir "cerebros industriosos" ${ }^{\prime \prime}$, sino una forma de saber entretejida a experiencias colectivas y ceremonias previas.

Por ello se aprende caminando, conversando, recordando y preguntando a los mayores.

Recorriendo territorio, "empalabrando" el caminar; observando y escuchando los leños ardientes de algún fogón; reconstituyendo lo dicho e indagando con preguntas que conllevan a tejer "la memoria colectiva de los antepasados". El pueblo Yanakona, organizado al interior de la UAIIN-CRIC, decía que:

El pensamiento yanakona cuestiona y va más allá de la razón, considera que la sabiduría es integral y tiene sentido a partir del sentir, pensar y actuar como un Pueblo. Por ello ante las

\footnotetext{
5 En Bolivia se creará la ciudad del conocimiento en Cochabamba; en Ecuador se ha creado otra ciudad del conocimiento denominado proyecto Yachay. Ambos proyectos tienen como finalidad cambiar la matriz productiva por la matriz de conocimiento. La base, el conocimiento industrial.
} 
disquisiciones racionales y lógicas debemos continuar sintiendo, pensando, actuando y trabajando.

Caminar, sudar la palabra, andarla con alegría, darle vida y fuerza nos ayuda a construir nuestra propia forma de entender y ver como pueblos indígenas. Despertar la memoria y seguir caminando tras las huellas de nuestros ancestros y hacer de la educación una herramienta política para seguir existiendo y perviviendo como pueblos indígenas, es nuestro sueño. (Cabildo Mayor Yanakona. 2008, p. 5.)

Caminar y sudar la palabra con alegría en un ritual como el Inti Raymi o en la visita ceremonial a lugares sagrados, implica que el mundo de la gente se está imbricando como un hilo que fluye a un tejido de mundos, ese mundo se constituye desde su interioridad como "red intra-mundo" que interacciona. La interacción tiene un sentido que, si bien implica compartir los planos de sabiduría en trascendencia, como decía el sabio de la Amawtay Wasi, Julio Sarancig (2013), implica también que el metabolismo de las acciones humanas no es una sensación atomizada en una experiencia personal 'exitosa' y gozosa de buenas energías ni el despliegue de un buen comportamiento depurado de alguna colonial culpa religiosa. La trascendencia implicaría una interacción, en tanto tiene una dimensión activa de apertura primordial a un tejido por tejer, por un hacer que se hace haciendo. El cuerpo, por tanto, es un nudo activo que dinamiza una red, entreteje diversas dimensiones constitutivas de múltiples flujos. Como dice Gerardo Simbaña, de la Amawtay Wasi, "imbrica existencias", por tanto, es un hacer/tejer orientado hacia todas las percepciones y existencias. El cuerpo entonces se imbrica a una determinada orientación de no sólo estar en el mundo sino estar, ser, conversar y fluir entre mundos. Por eso es importante en el mundo andino los encargos de abuelos, que funcionan como normas éticas de larga duración ancestral: "todo tiene vida", "todo en la vida es un ayni", "debes caminar mirando atrás, adelante y a los lados." (Yampara, 2016, p. 61).

La práctica de tejer la vida en una experiencia ritual también está asociada a la práctica del cultivo. Tejer y cultivar no son prácticas mecánico-productivas o un juego de imágenes artificiosas, guardan las sedimentaciones metafóricas de unas prácticas milenarias que se incluyen complementariamente. Por ello cuando el sabio aymara Yampara se refiere a "cultivar el Ayni" a lo largo de diversos tiempos-espacios de la Pacha, se refiere a la con-vivencia micro-política de un ritual con el tiempo-espacio extenso de los ciclos cósmicos agrícolas y sociales. Es cierto que este calendario cósmico entra en disputa con el calendario oficial y tiempo y "año colonial", como suele decir el sabio Saraguro, Fernando Sarango. De aquí la forma de vida, cómo uno vive las dimensiones del tiempo-espacio desde una perspectiva teleológica lineal o desde el qhip nayra (en aymara, el pasado está adelante, el futuro está atrás. Cómo uno se asume como runa, jaqi, nasa, yanakona u otra nominación milenaria o centenaria en ese ser y estar caminando la vida, pero también aprendiendo de las enseñanzas de los primeros padres y espíritus como el Aya Uma o Samay (en kichua), Ajayu (en aymara) o ksxa'w (en nasayuwe). En este sentido cabe preguntar por lo que significa la pluriversidad como experiencia y no sólo como dato descriptivo de una diversidad multicultural de pueblos.

\section{Conclusiones: el hilo y aliento de los ancestros}

Al culminar este entramado discursivo, se han expuesto algunos rasgos decoloniales de la Amawtay Wasi y la UAIIN-CRIC, como una respuesta del sentir/pensar/hacer propio a la colonialidad del poder, del saber, del ser y de la naturaleza. La luz de sabiduría de los sabios así como las prácticas rituales tensionan el binarismo dualista del conocimiento y la línea abismal jerarquizada sobre sujetos de conocimiento ancestral que se encuentran en la exterioridad de los muros y campus universitarios. Si la universidad convencional se ha asumido históricamente como la única centralidad de conocimiento, entronizando a científicos y expertos como los únicos sujetos de autoridad epistémica, sin negarlos, la UAlINCRIC y la Amawtay Wasi presentan otra opción de generación de conocimiento ligado al orden de la naturaleza y el cosmos. Desde la convivencialidad corporal fluye toda una poiesis integradora de la pluriversidad y el pluriverso de mundos. Esta dimensión de conocimiento en lugar territorial se opone a la destrucción capitalista y colonial de la vida y de la diversidad de mundos y tienden a reconstituir, como quizás diría Selnich Vivas (2015), "el hilo y aliento de los ancestros" (p.102). 


\section{Referencias}

Arnold, D., y De Dios, J. (2000). "Guerrero y tejedora: los thakhis del aprendizaje comunal". El rincón de las cabezas. Luchas textuales, educación y tierras en los Andes. La Paz: ILCA/FHCE.

Amawtay, W. (2004). Sumak Yachaipi, alli Kawsaypipash Yachakuna/ Aprender en la Sabiduría y el Buen Vivir/Learning Wisdom and the Good Way to Live. Quito: Gráficas AMZA.

Belaunde, L. (2008). El recuerdo de Luna, Género, sangre y memoria entre los pueblos amazónicos, 2da. Ed. CAAP en: Renjifo, G. (2012) "Interculturalidad y educación física en la escuela rural andina. Adiestrar el cuerpo para vencer en la escuela o criar el cuerpo para dialogar con ella", en: Diálogo de saberes. Una aproximación epistemológica. Lima: PRACTEC.

Cabildo, MY. (2011). Dar la palabra al territorio. Suyuma shimita kuy, El currículo espacio simbólico del territorio. Popayán: San Camilo.

(2008). Programa Educación. ¿La educación es el camino? Construyendo memoria Yanakona. Popayán: Litografía San José

CEFIC. (2007). Propuesta Pedagógica del Área Naturaleza y Sociedad. Popayán: PEBI.

(2004). ¿Qué pasaría si la escuela...? 30 años de construcción de una educación propia. Colombia: Fuego Azul.

Dávalos, P. (2013) De amawtas y philosophiae Doctor (PHD). La línea de fuego. 23 de abril.

De Sousa Santos, B. (2010). Descolonizar el saber, reinventar el poder. Montevideo: Trilce.

Escobar, A. (2016). Autonomía y diseño. La realización de lo comunal. Popayán: Editorial UC

(2014). Sentipensar con la tierra. Nuevas lecturas sobre desarrollo, territorio y diferencia. Medellín: Ediciones UNAULA.

Huanca, R. (2017). Tantthapit arunaka/Palabras juntadas. Ensayos sobre literatura y polifonía política. La Paz: THOA.

(2015). Las leyes de la Pacha, entre la proyección y la abyección del deseo: la interculturalidad de conocimientos y mundos en las leyes educativas de Ecuador y Bolivia. La Paz: THOA.

Lander, E. (2005). (Comp.). La colonialidad del saber: eurocentrismo y ciencias sociales, Perspectivas Latinoamericanas. Buenos Aires: CLACSO

Lambropoulos, V. (2005). The Rise of Eurocentrismo: Anatomy of Interpretation. Princeton, Princeton University Press. Citado en: Wallerstein, I. (2004). Las incertidumbres del saber. Barcelona: Gedisa.

Marcos, S. (2010). Cruzando fronteras: Mujeres indígenas y feminismos abajo y a la izquierda. Chiapas: Universidad de la Tierra.
Maya, D. (2014). “Revitalización de la historia de recuperación de tierra", en: López Adentro, Investigación y pensamiento indígena. Popayán: UAIIN-CRIC

Mignolo, W. (2014). "Educación y decolonialidad: aprender a desaprender para poder re-aprender", Un diálogo geopolíticopedagógico con Walter Mignolo, por: Facundo Giuliano y Daniel Berisso, en Revista IICE.

(2009). “Desobediencia Epistémica (II), Pensamiento Independiente y Libertad De-Colonial", en: Otros Logos, Revista de Estudios Críticos, Año 1 No. 1

Navia, W. (2002). Hermenéutica y comunicación. 2da. ed. La Paz: IEB.

Nene, G. "Pensamiento y acción pedagógica desde el camino del sol y la luna: avances, dificultades y retos para la implementación del calendario propio en la comunidad indígena de pueblo nuevo". Cxayu'ce. Por los caminos pedagógicos del SEIP, No. 19. Popayán: $\mathrm{PEBI} / \mathrm{CRIC}$

Quiiano, A. (2001). “Colonialidad del poder, cultura y conocimiento en América Latina", En: Walter Mignolo (comp.): Capitalismo y geopolítica del conocimiento. El eurocentrismo y la filosofía de la liberación en el debate intelectual contemporáneo. Buenos Aires: Ediciones del Signo.

(1999). "Colonialidad del poder, eurocentrismo y América Latina". Lima: Centro de Investigaciones Sociales.

Rizo, M. (2015). “Comunicación, cuerpo y subjetividad en Merleau Ponty", XXVII AMIC. Memorias. Facultad de Ciencias Políticas y Sociales. México, en: http://amic2015.uaq.mx.

Sarango, F. (2014). El paradigma educativo de Abya Yala, Continuidad histórica, avances y desafíos. Nicaragua: URACCAN.

(2008). Memorias. Primer Encuentro de Universidades Indigenas de Abya Yala. Quito: UTPL.

Saransig, P. (2013). Aportes al ordenamiento territorial desde la cosmovisión de los pueblos originarios, Pontificia Universidad católica del ecuador, Tesis de maestría PUCE, Quito, Ecuador.

SEIP, (2014). Sistema Educativo Indígena Propio. Popayán: PEBI-CRIC

Yampara, S. (2016). Suma qama qamaña. Paradigma Cosmo biótico Tiwanakuta. (Critica al sistema mercantil materialista). La Paz: Ediciones Qaman Qacha.

UINPI-AW, (2008). Memorias. Primer Encuentro de Universidades Indígenas de Abya Yala. UINPI_AW/CONESUP/COLAM/OUI-IOHE.

Viluche, J. (2011). Cxyu'ce. Programa de educación Bilingüe Intercultural PEBI-CRIC, No. 19, Popayán: CRIC-PEBI.

Vivas, S. (2015) "Jagagaiai manue uai: el narrar curativo", en: Komuya uai. Poética ancestral contemporánea, Colombia: GELCIL, Universidad de Antioquia 
Villavicencio, A. (2013). ¿Hacia dónde va el proyecto universitario de la Revolución Ciudadana? Quito: Taller Gráfico.

Walsh, C. (2009) Interculturalidad, estado, sociedad. Luchas (de) coloniales de nuestra época, Quito: Universidad Andina Simón Bolívar /Abya Yala.

Wallerstein, I. (2001), “El eurocentrismo y sus avatares: Los dilemas de la ciencia social", Walter Mignolo (comp.): Capitalismo y geopolítica del conocimiento. El eurocentrismo y la filosofía de la liberación en el debate intelectual contemporáneo. Buenos Aires: Ediciones del Signo.

Conversaciones con sabios, mayores y yachak

Mayora Dolly (Universidad Autónoma Indígena Intercultural-Consejo Regional Indígena del Cauca- UAIIN-CRIC)

Gerardo Simbaña (Universidad Indígena Intercultural Amawtay Wasi)

Benigno Shingre (Universidad Indígena Intercultural Amawtay Wasi) Fernando Sarango (Universidad Indígena Intercultural Amawtay Wasi)

Jaime Pilatuña (Universidad Indígena Intercultural Amawtay Wasi)

José María Vacacela (Universidad Indígena Intercultural Amawtay Wasi)

Gonzalo Atto (Universidad Indígena Aymara Boliviana Tupak Katari)

Joaquín Viluche (Universidad Autónoma Indígena InterculturalConsejo Regional Indígena del Cauca- UAIIN-CRIC)

Manuel Sisco (Universidad Autónoma Indígena Intercultural-Consejo Regional Indígena del Cauca- UAIIN-CRIC)

Smith Colque (Universidad Indígena Boliviana Aymara Tupak Katari) 